\title{
Evidence for Non-Gaussian Tail in 3-Dimensional Pion Emission Source at SPS
}

\author{
P. Chung ${ }^{1}$ and P. Danielewicz ${ }^{2}$ for The NA49 Collaboration \\ 1 Dept of Chemistry, SUNY Stony Brook, Stony Brook, NY 11794, USA \\ 2 National Superconducting Cyclotron Laboratory and Department of Physics and Astronomy, \\ Michigan State University, East Lansing, MI 48824-1321, USA
}

Received on 2 November, 2006; Revised version on 29 March, 2007; Third version on 12 April, 2007

\begin{abstract}
The NA49 experiment at CERN SPS has acquired a huge data set of $\mathrm{Pb}+\mathrm{Pb}$ events over a broad range of energy and centrality during the last several years. This high statistics data set, coupled with a state-of-the-art analysis technique, allows for the first model-independent extraction and energy scan of 3D emission sources for pion pairs at SPS energies. These 3D pion emission sources provide new insights into the nature of a long-range source previously reported by PHENIX at RHIC. Preliminary results indicate that the pion source function is essentially Gaussian from $20 \mathrm{AGeV}$ to $80 \mathrm{AGeV}$ but it displays significant non-Gaussian tails at $158 \mathrm{AGeV}$.
\end{abstract}

Keywords: Correlation; Interferometry; Intensity interferometry; Cartesian harmonics; Anisotropic correlation

\section{INTRODUCTION}

A deconfined phase of nuclear matter is expected to be formed at the high energy densities created in relativistic heavy ion collisions [1]. It is widely believed that important signatures of such a phase are reflected in the space-time extent and shape of particle emission source functions.

Recently, 1-Dimensional source imaging techniques [2,3] have revealed a non-trivial long range structure in the twopion emission source at RHIC [4,5]. The origins of this structure are still unclear. The presence/absence of such a structure in the pion emission sources in heavy ion collisions at intermediate SPS energies could yield important information which could help resolve the structure's origins. The NA49 Collaboration has carried out $\mathrm{Pb}+\mathrm{Pb}$ collisions over a wide range of bombarding energies at the CERN SPS during the last decade [6]. Such a rich data set provides a unique opportunity to search for this long range structure at the SPS and study its evolution with beam energy with a view to unraveling its nature.

In this paper, preliminary $3 \mathrm{D}$ emission source images for pions produced in central $\mathrm{Pb}+\mathrm{Pb}$ collisions over the incident energy range 20 to $158 \mathrm{AGeV}$ are presented. The results are discussed in the context of a Gaussian shape assumption.

\section{EXPERIMENTAL SETUP AND DATA ANALYSIS}

The data presented here were taken by the NA49 Collaboration during the years 1996-2002. The incident beams of 20, 80, 158 AGeV were provided by the CERN SPS accelerator. The NA49 Large Acceptance Hadron Detector [7] achieves large acceptance precision tracking $\left(\delta p / p^{2} \approx(0.3-\right.$ 7). $10^{-4}(\mathrm{GeV} / \mathrm{c})^{-1}$ [7]) and particle identification using time projection chambers. Charged particles are detected by the tracks left in the TPC and identified by the energy deposited in the TPC gas. Mid-rapidity particle identification is further enhanced by a time-of-flight wall (resolution 60 ps). Event centrality is determined by a forward calorimeter which measures the energy of spectator matter.

$3 \mathrm{D}$ correlation functions, $\mathrm{C}(\mathbf{q})$, were obtained as the ratio of pair to uncorrelated reference distributions in relative momentum $\mathbf{q}$ for $\pi^{-} \pi^{-}$pairs. Here, $\mathbf{q}=\frac{\left(\mathbf{p}_{1}-\mathbf{p}_{2}\right)}{2}$ is half of the relative momentum between the two particles in the Pair Center-ofMass System (PCMS) frame. The pair distribution was obtained using pairs of particles from the same event and the uncorrelated distribution was obtained by pairing particles from different events.

Track merging and splitting effects were removed by appropriate cuts on both the pair and uncorrelated distributions. The pair cuts require the two particles in each pair to be separated by at least $2.2 \mathrm{~cm}$ over 50 pad rows in the main vertex TPC. A $20 \%$ increase in this minimum separation results in the correlation data points fluctuating within the statistical errors. Hence, the systematic uncertainty associated with the pair cuts is deemed smaller than the statistical uncertainty.

The effects of track momentum resolution were assessed by jittering the momentum of the tracks in the data by the maximum resolution, $\delta p / p^{2} \approx 7.10^{-4}(\mathrm{GeV} / \mathrm{c})^{-1}$ [7]. The resulting re-computed $3 \mathrm{D}$ correlation function incorporates twice the effect of the momentum resolution and was found to be identical to the raw un-smeared correlation function for incident beam energies up to $80 \mathrm{AGeV}$. This is not surprising, considering that the mean momentum of the low $p_{T}$ tracks used is $1.2 \mathrm{GeV} / \mathrm{c}$, resulting in a momentum resolution of $\delta p / p \approx\left(1.2 \times 7.10^{-4}\right)=0.1 \%$. Such a small momentum resolution has no observable effect on the correlation function.

At $158 \mathrm{AGeV}$, the above smearing procedure results in the correlation peak in the region $q<10 \mathrm{MeV}$ to be slightly smeared. This tends to reduce the imaged source intensity at large radii. Hence, the preliminary results presented here would be a lower limit of the actual source function.

In the cartesian harmonic decomposition technique [8, 9], the $3 \mathrm{D}$ correlation function is expressed as

$$
f C(\mathbf{q})-1=R(\mathbf{q})=\sum_{l} \sum_{\alpha_{1} \ldots \alpha_{l}} R_{\alpha_{1} \ldots \alpha_{l}}^{l}(q) A_{\alpha_{1} \ldots \alpha_{l}}^{l}\left(\Omega_{\mathbf{q}}\right)
$$

where $l=0,1,2, \ldots, \alpha_{i}=x, y$ or $z, A_{\alpha_{1} \ldots \alpha_{l}}^{l}\left(\Omega_{\mathbf{q}}\right)$ are cartesian harmonic basis elements $\left(\Omega_{\mathbf{q}}\right.$ is solid angle in $\mathbf{q}$ space) and $R_{\alpha_{1} \ldots \alpha_{l}}^{l}(q)$ are cartesian correlation moments given by

$$
R_{\alpha_{1} \ldots \alpha_{l}}^{l}(q)=\frac{(2 l+1) ! !}{l !} \int \frac{d \Omega_{\mathbf{q}}}{4 \pi} A_{\alpha_{1} \ldots \alpha_{l}}^{l}\left(\Omega_{\mathbf{q}}\right) R(\mathbf{q})
$$


The coordinate axes are oriented so that $z$ is parallel to the beam (long) direction, $x$ points in the direction of the total momentum of the pair in the LCMS frame (out) and $y$ is perpendicular to the other two axes (side).

The correlation moments, for each order $l$, are calculated from the measured 3D correlation function using Eq. (2). Each independent correlation moment is then imaged using the 1D Source Imaging code of Brown and Danielewicz [2, 3] to obtain the corresponding source moment for each order $l$. Thereafter, the total source function is calculated by combining the source moments for each $l$ as in Eq. (3)

$$
S(\mathbf{r})=\sum_{l} \sum_{\alpha_{1} \ldots \alpha_{l}} S_{\alpha_{1} \ldots \alpha_{l}}^{l}(r) A_{\alpha_{1} \ldots \alpha_{l}}^{l}\left(\Omega_{\mathbf{r}}\right)
$$

\section{RESULTS}

Figure 1 shows the $l=0\left(R^{0}\right)$ and $l=2\left(R_{x x}^{2}\right.$ and $\left.R_{y y}^{2}\right)$ moments for mid-rapidity $\left(\left|y_{L}-y_{0}\right|<0.35\right.$, where $y_{L}$ is particle laboratory rapidity and $y_{0}$ is $\mathrm{CM}$ rapidity), low $p_{T}(0.0<$ $\left.p_{T}<0.2 \mathrm{GeV} / \mathrm{c}\right) \pi^{-} \pi^{-}$pairs from $20 \mathrm{AGeV}$ central $(7 \%)$ $\mathrm{Pb}+\mathrm{Pb}$ collisions as a function of the relative momentum $\mathrm{q}$ in the PCMS frame. Higher order moments are either zero or negligible. The Lorentz transformation of $\mathbf{q}$ from the laboratory frame to the PCMS is done by a Lorentz transformation to the pair Locally Co-Moving System frame (LCMS) along the beam direction followed by a transformation to the PCMS along the direction of transverse momentum of the pair.

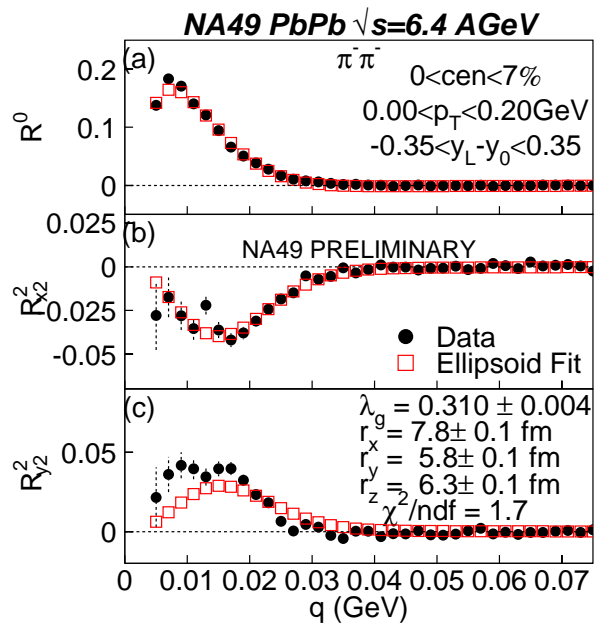

FIG. 1: (a) $l=0$ and $l=2$ ((b) and (c)) moments for mid-rapidity low $p_{T} \pi^{-} \pi^{-}$pairs from $20 \mathrm{AGeV}$ central $\mathrm{Pb}+\mathrm{Pb}$ collisions as a function q. Data are shown as solid circles while the squares represent the result of a simultaneous fit of the moments with an ellipsoid shape.

The data points are shown in solid circles and the open squares represent the result of a simultaneous fit to the independent moments with a ellipsoid shape (3D Gaussian). The fit parameters are shown in the bottom panel. The extracted

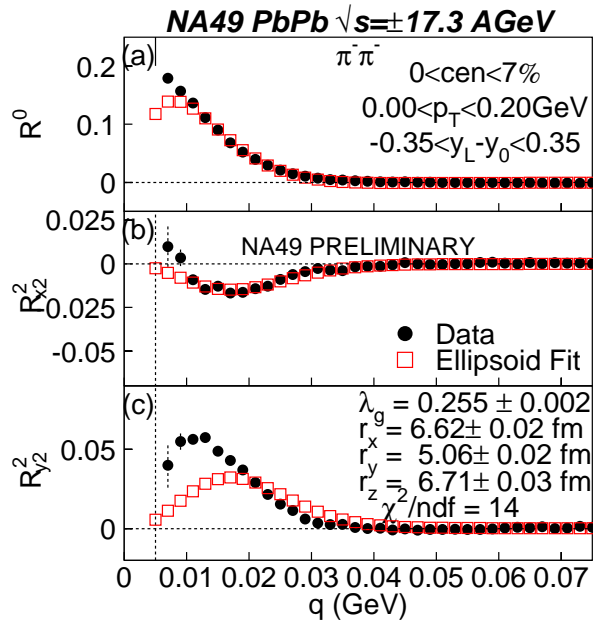

FIG. 2: Same as Fig.1 but for $158 \mathrm{AGeV} \mathrm{Pb}+\mathrm{Pb}$ collisions.

data moments are fairly well represented by the Gaussian shape.

Figure 2 shows the corresponding moments for $\pi^{-} \pi^{-}$pairs from $158 \mathrm{AGeV} \mathrm{Pb}+\mathrm{Pb}$ collisions. In this case, the fit result shows that the data moments are not adequately described by the Gaussian assumption: $R^{0}$ and $R_{y 2}^{2}$ exhibit significant discrepancies between the data and the fit moments.

For the low $p_{T}$ pairs considered, the only significant source moments are found for multipolarities $l=0$ and 2. Hence, the total source functions in the $x, y$ and $z$ directions are simply the sum of the 1D source $S^{0}$ and the corresponding $l=2$ moment $S_{i i}^{2}$ where $\mathrm{i}=x, y$ or $z$. Fig. 3(a)-(c) shows the total

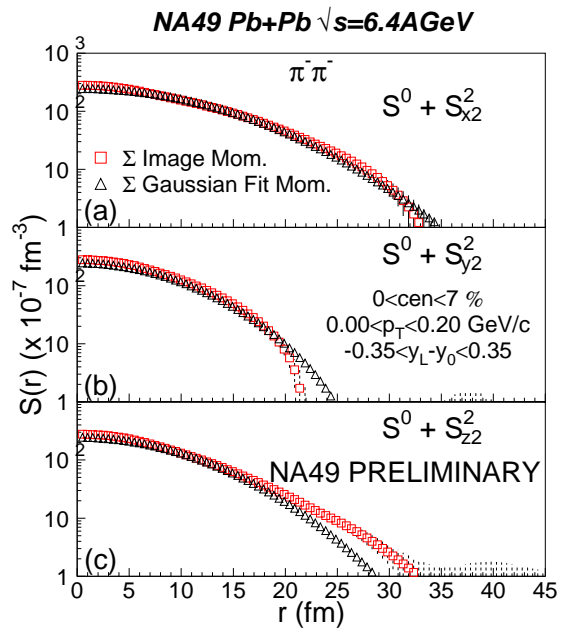

FIG. 3: Total source function in (a) $x$ (out) (b) $y$ (side) (c) $z$ (long) direction for low $p_{T}$ mid-rapidity $\pi^{-} \pi^{-}$pairs from central $\mathrm{Pb}+\mathrm{Pb}$ collisions at $\sqrt{s}=6.4 \mathrm{AGeV}$ (Incident beam energy $=20 \mathrm{AGeV}$ ). Squares represent source image while triangles represent Gaussian assumption fit function result.

source function for mid-rapidity, low $p_{T}$ pion pairs from central $\mathrm{Pb}+\mathrm{Pb}$ collisions at $20 \mathrm{AGeV}$ (i.e $\sqrt{s}=6.4 \mathrm{AGeV}$ ). The source image is shown in squares while the triangles represent an ellipsoid (3D Gaussian) shape fit to the 3D correlation 
function. The source image and the Gaussian fit agree reasonably well in all 3 directions.

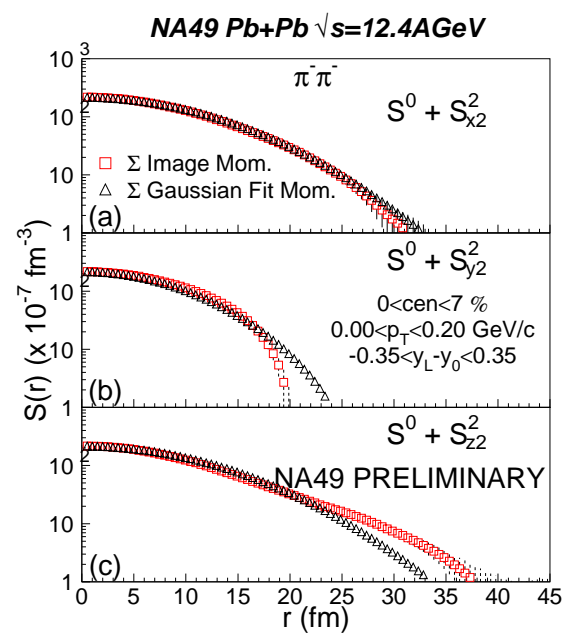

FIG. 4: Same as Fig.3 but for $\sqrt{s}=12.4 \mathrm{AGeV}$ (Beam energy $=$ $80 \mathrm{AGeV})$.

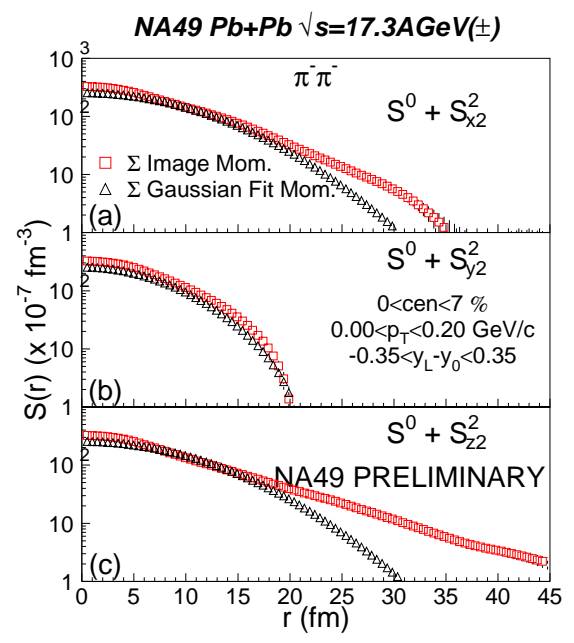

FIG. 5: Same as Fig.3 but for $\sqrt{s}=17.3 \mathrm{AGeV}$ (Beam energy $=$ $158 \mathrm{AGeV}$ ). Result is average over runs with +ve and -ve magnetic field.
The same conclusion is reached from Fig. 4(a)-(c) for low $p_{T}$ pion pairs from central $\mathrm{Pb}+\mathrm{Pb}$ collisions at $80 \mathrm{AGeV}$ $(\sqrt{s}=12.4 \mathrm{AGeV})$ respectively.

However, at $158 \mathrm{AGeV}$ incident energy $(\sqrt{s}=17.3 \mathrm{AGeV})$, Fig. 5(a),(c) show that there are significant deviations of the source image from the Gaussian fit in the $x$ and $z$ directions respectively: the source image seems to exhibit a non-Gaussian tail which extends beyond the range of the Gaussian fit for $r>17 \mathrm{fm}$ in the out and long directions.

\section{DISCUSSION}

The evolution of the pion source image with increasing beam energy indicates that the source function is essentally Gaussian from $20 \mathrm{AGeV}$ to $80 \mathrm{AGeV}$ but it displays very prominent non-Gaussian tails at $158 \mathrm{AGeV}$ in the out and long directions. Hence, the long range structure, first observed at RHIC $(\sqrt{s}=200 \mathrm{AGeV})$ [5], is already manifest at much lower energies at the SPS. This points to the expectation that there are new physics processes which set in between $80 \mathrm{AGeV}(\sqrt{s}=12.4 \mathrm{AGeV})$ and $158 \mathrm{AGeV}$ $(\sqrt{s}=17.3 \mathrm{AGeV})$ which result in the non-Gaussian tails in this energy regime.

Could the non-Gaussian tails observed at $158 \mathrm{AGeV}$ be due to resonance decays such as the $\omega$ meson? Although a quantitative issue, the resonance population does not change significantly from $\sqrt{s}=12.4 \mathrm{AGeV}$ to $\sqrt{s}=17.3 \mathrm{AGeV}$. Hence, it is unlikely that resonance decays could account for the observed non-Gaussian tail at $158 \mathrm{AGeV}$.

\section{v. CONCLUSIONS}

The NA49 Collaboration has performed the first extraction of emission source functions for mid-rapidity, low $p_{T}$ pions produced in central $\mathrm{Pb}+\mathrm{Pb}$ collisions over the beam energy range 20-158 AGeV at SPS. Preliminary results indicate that the source functions are essentially Gaussian from 20 to $80 \mathrm{AGeV}$. However, at $158 \mathrm{AGeV}$ the pion source function displays non-Gaussian tails in the out and long directions. It is unlikely that these non-Gaussian tails originate from secondary pions produced in resonance decays.
[1] Quark Matter 2005 Proceedings of the 18th International Conference on Ultra-Relativistic Nucleus-Nucleus Collisions, Budapest, Hungary, Aug. 4-9, 2005, Nucl. Phys. A 774 (2006).

[2] D.A. Brown and P. Danielewicz, Phys. Lett. B 398, 252 (1997).

[3] D.A. Brown and P. Danielewicz, Phys. Rev. C 57, 2474 (1998).

[4] P. Chung et al., Nucl. Phys. A 749, 275c (2005).

[5] S.S. Adler et al. (PHENIX Collaboration), Phys. Rev. Lett., 98, 132301 (2007).

[6] C. Alt et al. (NA49 Collaboration), CERN-SPSC-2005-041,
CERN-SPSC-P-264-ADD-12, Nov 2005.

[7] S. Afanasiev et al., Nucl. Instrum. Meth. A 430, 210 (1999).

[8] P. Danielewicz and S. Pratt, Phys. Lett. B 618, 60 (2005).

[9] P. Chung et al., XXXV International Symposium on Multiparticle Dynamics, Kroměříž, Czech Republic, 9-15 August 2005 and Workshop on Particle Correlations and Femtoscopy, 15-17 August 2005, AIP Conf. Proc. 828, 499 (2006). 